\title{
Innovative uses of existing software in the teaching of psychodiagnostics
}

\author{
CHRIS E. STOUT \\ Forest Hospital and Foundation, Des Plaines, Illinois \\ and \\ MYCHAIL SCHERAMIC \\ Forest Institute, Des Plaines, Illinois
}

\begin{abstract}
Too frequently, current, adaptable software programs are not used to their full potential for educational and clinical applications. Graduate psychology students often become familiar with computers and various programs for word processing and research applications, and now these students, using the same systems, can utilize and adapt database software to assist in psychodiagnostic decision-making. This paper discusses the development of one such system.
\end{abstract}

This paper deals with the application of database software to the teaching of psychodiagnostic decision-making abilities. In past works (Hirshfeld, Spitzer, \& Miller, 1974; Overall \& Hollister, 1964; Spitzer \& Endicott, 1968; Stout, 1988), diagnostic programs often were based on sophisticated programs that were difficult to use, were not user-friendly, or did not provide adequate descriptors. This paper discusses how existing, widely used software can, and was adapted to, teach the frequently difficult process of psychodiagnostic judgment. The American Psychiatric Association's 1987 edition of Diagnostic and Statistical Manual of Mental Disorders (DSM-III-R) provides a specialized index of selected symptoms (compiled by M. B. First and R. L. Spitzer, pp. 519-552). This symptom index is not intended (by design of the authors) to be the basis for making a differential diagnosis, but rather to act as a heuristic aid in identifying "those diagnostic criteria in the various disorders that refer, either precisely or in a more general way, to a particular symptom" (p. 519). This symptom index then was automated, or computerized, within a cross-referenced, descriptive, and deductive database system. Symptoms in this system are categorized into 15 broad areas: Activity, Anxiety Symptoms, Appearance, Behavior, Cognition/ Memory/Attention, Eating Disturbance, Energy, Form and Amount of Thought/Speech, Mood/Affect Disturbance, Occupational and Social Impairment, Perceptual Disturbance (including Hallucinations), Personality Traits, Physical Signs and Symptoms, Sleep Disturbance, and Thought Content (including Delusions) (American Psychiatric Association, 1987).

Correspondence may be addressed to Chris E. Stout, Psychodiagnostics Division, Forest Hospital and Foundation, 555 Wilson Lane, Des Plaines, IL 60016.
Many theoreticians and clinicians view accurate psychodiagnosis as the initial component necessary in appropriate, ethical treatment (Bishop, Sharf, \& Adkins, 1975; Hurst, Weigel, Thatcher, \& Hyman, 1969; Johnson, 1965). Often, however, theoretical biases of professors disrupt the more scientific teaching of psychodiagnostic methods (e.g., what symptoms to look for, what type of questions to ask, etc.). DSM-III-R is atheoretical in its presentation of symptom clusters and diagnostic classifications. This is not to say that the DSM series is without fault or critics; however, it is certainly the most widely used and accepted methodology of diagnostic nosology that we currently have. Its automation of symptomindexing aids to further distance the contamination of a clinician's or instructor's biases.

Computerization of mental health services is widespread: automated testing and report writing; automated, branching histories that are computer-patient interactive; word processing systems that follow certain stylistic conventions; computerized billing and accounting systems; expert systems and artificial intelligence applications for prediction models; advanced statistical analyses; and more. Yet, little attention is paid to the instructional value of current systems or to the teaching of the various uses of preexisting software. There needs to be further development and integration in this virtually ignored area. This system and methodology is intended to be a model towards a solution.

Although many individuals are becoming computer literate, this does not always mean that they are able to program or understand various programming languages. What such individuals do learn is how to specifically run common types of software packages; especially those with "help" functions, or windows, that are user-friendly. We chose to utilize Ashton-Tate's dBase III + database software package because it is readily available, very popu- 
lar and is already used in many sites, has helpful windows and a menu-driven mode that are very user-friendly, requires no user programming language abilities, has good user support, and is quite powerful. Thus, the students who have learned dBase III + in a research course now can use the same commands and the same program in clinical, psychodiagnostic settings without having to contend with new, alien software. This, in and of itself, can be important in decreasing the anxiety associated with learning an entire diagnostic nosology.

This system was designed to be utilized in a variety of ways. It was intended to aid students (or professionals) in arriving at a psychodiagnostic classification based on the patient's presentation of symptoms, but it also can be used to define psychiatric symptomology terms, employ cross-referential diagnostic numbers to diagnostic categories, and even refer the user to a specific page number in $D S M-I I I-R$ for further reference. The data file strictly follows $D S M-I I I-R$ criteria and thus parallels the accuracy of the DSM.

A differential psychodiagnostic program (Stout, 1988) was helpful for students and professionals; however, some individuals were unable to get the program up and running in BASIC. And yet, some of these same individuals could effortlessly handle this dBase III + version. This version also allowed for symptom clusters to be input, whereas, with the prior work, this had not been possible.

An additional development is that a student may also utilize a "crib sheet" of symptoms to which they can refer during a patient interview. Again, this was adapted from $D S M-I I I-R$ to be accurate and consistent with the method of patient symptom classification. Since the database offers a number of helpful features (symptom definitions, page number references for additional information, etc.) as options, experienced diagnosticians may choose not to utilize them or may choose to reference them to doublecheck their perceptions or refresh their memories.

This database will run on any IBM PC or on most IBMcompatibles with two 640K disk drive that can run AshtonTate's dBase III +. ( A 10-Mb hard drive is more convenient but not necessary.) No programming knowledge is necessary, only some basic familiarity with dBase III+ and psychiatric symptomology. If one needs help, the dBase III + manual or other similar handbooks are readily available at most book or computer stores.

This program is not intended to be a substitute for a full or partial psychological assessment. Its data (symptom) collection form helps to standardize patient data collection and can be used as an interview and diagnostic guide for rapid and thorough symptom examination. The form is meant to be used as a basis from which the clinician can branch out questions in order to stimulate or further enhance data collection.

The database file that is used with $\mathrm{dBase}$ III + database can be loaded onto a hard disk or onto a double-sided, double-density $5^{1 / 4}$-in. floppy disk. To load the file onto a hard disk, the user can refer to the standard MS-DOS commands to copy the disk (COPY*.*).

The database is opened via the standard command for dBase III + (C > "DBASE'). Upon seeing the prompt, the user types in USE DSM.DBF to open the file. Once the database is opened, the user finds the traditional pulldown menus, which can be used in numerous ways in creating searches by pressing the $\mathrm{F} 2$ key or by typing in ASSIST (and one return). For example, if the user wishes to create a search to find diagnoses associated with certain symptoms, he/she would use the RETRIEVE menu to activate the choice marked LIST. Next, the user would choose BUILD A SEARCH CONDITION, which would enable him/her to enter in the names (or assigned numbers) of the symptoms in question.

The database can be used to find the definitions for symptoms, fifth-digit classification meanings, DSM-III$R$ code numbers, and diagnostic classifications (e.g., Organic Mental Disorders). Diagnoses may be entered and a listing of associated symptoms may be retrieved. The ways in which the database may be utilized is limited only by the user's imagination. Users may modify the database's structure and add to or delete records to further enhance the database to meet their particular needs and interests, or users can directly type in commands at the "." prompt (e.g., LIST SYMPTOM, DIAGNOSIS FOR SX_NUM $=\mathrm{XX} . \mathrm{OR} . \mathrm{SX} \_\mathrm{NUM}=\mathrm{YY}$ [return]).

Readers interested in the program should write to the primary author.

\section{REFERENCES}

american Psychiatric Association (1987). Diagnostic and statistical manual of mental disorders (3rd ed.-rev.). Washington, DC: Author.

Bishop, J. B., ShaRF, R. S., \& Adkins, D. M. (1975). Counselor intake judgments, client characteristics, and number of sessions at a university counseling center. Journal of Counseling Psychology, 22, 557-559.

HirshFeld, R., Spitzer, R. L., \& Miller, R. G. (1974). Computer diagnosis in psychiatry: A bayes approach. Journal of Nervous \& Mental Diseases, 158, 339-407.

Hurst, J. C., Weigel, R. G., Thatcher, R., \& Hyman, A. J. (1969). Counselor-client diagnostic agreement and perceived outcome of counseling. Journal of Counseling Psychology, 16, 421-426.

Johnson, R. W. (1965). Number of interviews, diagnosis, and success of counseling. Journal of Counseling Psychology, 12, 248-251.

Overall, J. E., \& Hollister, L. E. (1964). Computer procedures for psychiatric classification. Journal of the American Medical Association, 18, 583-585.

SPITZER, R. L., \& ENDICotT, J. (1968). DIAGNO: A computer program for psychiatric diagnosis utilizing a differential diagnostic procedure. Archives of General Psychiatry, 18, 746-796.

STout, C. E. (1988). Personal computer software for teaching differential psychodiagnostics. Behavior Research Methods, Instruments, \& Computers, 20, 106-107. 